\title{
A Review of Rapid Distortion Theory
}

\section{Sudipta Lal Basu ${ }^{1, a}$, Breiffni Fitzgerald ${ }^{1, b}$, Søren R.K.Nielsen ${ }^{2, c}$ and Biswajit Basu ${ }^{1, \mathrm{~d}^{*}}$}

${ }^{1}$ Dept. of Civil, Structural \& Environmental Engineering, Trinity College Dublin, Dublin, Ireland

${ }^{2}$ Department of Civil Engineering, Aalborg University, Denmark

abasus@tcd.ie, bBreiffni.Fitzgerald@tcd.ie, 'srkn@civil.aau.dk, dbasub@tcd.ie*

* corresponding author

\section{Keywords: Rapid Distortion Theory, Turbulence, Wind Energy, Wind Farms}

\begin{abstract}
With the depleting non-renewable fuel sources like coal and an ever-increasing demand for energy, we need to start looking into renewable energy sources. These are of paramount importance for a sustainable and green future. Wind Energy is one of the most important sources of renewable energy. But, setting up a wind farm requires considerable land area and land acquisitions are often faced with legal hurdles. This necessitates setting up offshore wind turbines. But, when we talk about offshore wind farms, we need to address the age-old phenomenon: "Turbulence". Presently, we are trying to develop enhanced controllers for wind farms which will increase the efficiency of the wind farms. The effects of rapidly changing wake aerodynamics i.e. breakdown of strong tip and hub vortices mixed up with low intensity turbulence in the inflow of the rotor and counter-rotation of the wake i.e. determinate velocity component in wake turbulence field will affect the overall performance of the wind farm. This paper provides a brief review on Rapid Distortion Theory (RDT) to model the turbulence.
\end{abstract}

\section{Introduction}

Batchelor, in European Turbulence Conference, 1986 at Lyon predicted that there could be no global theory for turbulence (other than that turbulent flows are governed by Navier-Stokes equations) because all turbulent flows are governed by the initial and boundary conditions. Batchelor \& Proudman [1] had given a description of how turbulence is distorted when it passes rapidly through a region where large-scale straining motions are induced.

This review paper gives a brief description of the classification of the different types of problems of turbulence, a brief overview of the RDT and the errors associated with it. The application of the RDT to model the turbulence in case of offshore wind farms is also presented.

\section{Classification of Turbulence}

Hunt and Carruthers [4] in their paper "Rapid Distortion Theory and 'problems' of turbulence" have classified the turbulent flows based on their initial and boundary conditions.

\section{Class I: Closed domains and deterministic boundary conditions}

In this case, boundary surface of the domain consists of rigid stationary or moving surfaces. Turbulent motion could be induced due to the motion of the boundaries or due to the body forces. Example can be taken of a cylinder with a moving piston. 


\section{Class II: Open domains and statistical boundary conditions}

In this class of turbulent flows, some, if not all of, the bounding surfaces of the domain lie within the fluid itself. Let $\mathscr{E}$ denote the region outside domain $\mathscr{Y}$. An example can be of the turbulence in the wake of the wind farm itself. This is further subdivided into:-

i. No turbulence in $\mathscr{E}:$ In this class the flow enters $\mathscr{P}$ with characteristic mean velocity $\mathrm{U}_{0}$ and the turbulence could be generated due to instabilities if Reynolds Number is high.

ii. Turbulence in $\mathscr{E}$ with significant mean flow from $\mathscr{C}$ to

iii. Turbulence in $\mathscr{E}$ without significant mean flow from $\mathscr{E}$ to $\mathscr{O}$

\section{Class III: Initial conditions and changing boundary conditions}

In both Class I and II above, the nature of turbulence is dependent on the boundary of the domain but with the assumption that the boundary conditions persist for long enough or they do not change that rapidly to cause an ongoing time-dependent change at $t=\tau$, $\tau$ being the instantaneous time at which we are interested in the nature of turbulence. This third classification considers the change in boundary conditions.

\section{Mathematical developments of RDT}

The linearization and the error analysis presented in this paper are from the works of Hunt and Carruthers [4]. The results of the same are studied and an effort will be made to implement the same in the case of wind farms. In the equations discussed, notations in bold indicate vector quantities.

\section{Linearization}

Reynolds suggested that the random velocity, pressure and vorticity fields $\mathbf{u}(\mathbf{x}, t), \mathbf{p}(\mathbf{x}, t), \boldsymbol{\omega}(\mathbf{x}, t)$ as functions of position vector $\mathbf{x}$ and time, $t$ can be divided into components of ensemble mean and fluctuating component i.e. $\mathbf{u}=\mathbf{U}+\mathbf{u}^{\prime}, \mathbf{p}=\rho\left(\mathbf{P}+\mathbf{p}^{\prime}\right), \boldsymbol{\omega}=\boldsymbol{\Omega}+\boldsymbol{\omega}^{\prime}$ where $\mathbf{U}, \mathbf{P}, \boldsymbol{\Omega}$ are the ensemble means and $\mathbf{u}^{\prime}, \mathrm{p}, \boldsymbol{\omega} \boldsymbol{\omega}^{\prime}$ are the fluctuating components. Discussion in this paper is strictly confined to incompressible flows in the absence of body forces. The governing equations for velocity and vorticity (in Einstein notation) are

$$
\begin{aligned}
& \frac{\partial u_{i}^{\prime}}{\partial t}+U_{j} \frac{\partial u_{i}^{\prime}}{\partial x_{i}}+u_{j}^{\prime} \frac{\partial U_{i}}{\partial x_{j}}=-\frac{1}{\rho} \frac{\partial p^{\prime}}{\partial x_{j}}+v \nabla^{2} \mathrm{u}_{i}^{\prime}-(\mathrm{NL})_{u_{i}^{\prime}{ }^{\prime} \cdots} \\
& \frac{\partial \omega_{i}^{\prime}}{\partial t}+U_{j} \frac{\partial \omega_{i}^{\prime}}{\partial x_{j}}+u_{k}^{\prime} \frac{\partial \Omega_{i}}{\partial x_{k}}-\omega_{k}^{\prime} \frac{\partial U_{i}}{\partial x_{n}}-\Omega_{n} \frac{\partial u_{i}^{\prime}}{\partial x_{n}}=v \nabla^{2} \omega_{i}^{\prime}+(\mathrm{NL})_{\omega^{\prime} i} \cdots
\end{aligned}
$$

where, $v=$ kinematic viscosity, $\partial u_{i}^{\prime} / \partial x_{i}=0, \omega_{i}^{\prime}=\varepsilon_{i j k} \partial u_{k}^{\prime} / \partial x_{j}$ so that $\partial \omega_{k}^{\prime} / \partial x_{k}=0$ The nonlinear terms in the above equations are

$$
(\mathrm{NL})_{u \prime i}=-\left[\frac{\partial\left(u_{k}^{\prime} u_{i}^{\prime}\right)}{\partial x_{k}}-\frac{\partial\left(\overline{u_{k}^{\prime} u_{i}^{\prime}}\right)}{\partial x_{k}}\right] \ldots
$$




$$
(\mathrm{NL})_{\omega \prime i}=-u_{k}^{\prime}{ }_{k} \frac{\partial \omega_{i}^{\prime}}{\partial x_{k}}+\omega_{j}^{\prime}{ }_{j} \frac{\partial u_{i}^{\prime}}{\partial x_{j}}+\frac{\overline{u_{k}^{\prime} \partial \omega^{\prime}}}{\partial x_{j}}-\frac{\overline{\omega_{j}^{\prime} \partial u_{l}}}{\partial x_{j}} \ldots
$$

To linearize the governing equations, the nonlinear terms (NL) are to be ignored. An effort is then required to be made to find a solution for the linearized form of the equations by applying suitable boundary conditions. However, ignoring the (NL) terms will result in errors which in turn needs to be analysed so that solution of the linearized equation is acceptable and reflects the nearly acceptable physical scenario of the problem, if not exact.

While dealing with offshore wind-turbines, the mean velocity of the wind is significantly higher compared to its fluctuations. Apart from ignoring the (NL) terms, for air kinematic viscosity is of the order of $10^{-5} \mathrm{~m}^{2} / \mathrm{s}$ and we can infer that viscosity will not have much effect on the final result, so it will be negligible and can be ignored. Thus, we can view this problem as part of ClassII(Type-ii) case of classification stated above.

\section{Error Analysis}

In order to estimate the error associated with the linearization, we begin with assuming a typical r.ms. velocity $u_{0}^{\prime}=\left(\frac{1}{3} \overline{u_{\imath} u_{\imath}}\right)^{1 / 2}$ and integral scale $L_{x}$ for the large energy containing scales of turbulence and for small eddies with velocity scale $\mathrm{u}(l)$ and length scale $l$. $\mathrm{U}_{0}$ and $\Delta \mathrm{U}_{0}$ are the typical values of the mean velocity and the change in mean velocity respectively over a typical length scale in 9 . The two-point moment of velocity field, $R_{i j}(\boldsymbol{r})=\overline{u_{l}^{\prime}(\boldsymbol{x}) u_{j}^{\prime}(\boldsymbol{x}, \boldsymbol{r})}$ or the twopoint structure function, $\Delta R_{i i}=\overline{\left(u_{l}(\boldsymbol{x})-u_{l}(\boldsymbol{x}, \boldsymbol{r})\right)^{2}}$ ( $\boldsymbol{r}$ is the vector defining distance between the two points) are primarily calculated from the linearized equations. If $\omega$ ' is used to calculate $\mathbf{u}$ ' and $R_{\mathrm{ii}}$, the conditions for linearization are different from using $\overline{\omega^{\prime}}{ }_{l}^{2}$ because of the requirement of specifying the scale of vorticity field contributing to the moment.

Batchelor [5], using Biot-Savart integral showed that $\Delta R_{i i}(\boldsymbol{x}, \boldsymbol{r})$ can be expressed as an integral of $\overline{\omega^{\prime}{ }_{k}\left(\boldsymbol{r}^{\prime}\right) \omega^{\prime}{ }_{l}\left(\boldsymbol{r}^{\prime \prime}\right)}$, where $\boldsymbol{r}^{\prime}$ and $\boldsymbol{r}^{\prime \prime}$ are the displacement vectors corresponding to $\mathrm{k}$ and 1 respectively. For high Reynolds number it can be expressed in terms of rate of dissipation per unit mass, $\varepsilon$ by the following relation

$$
\Delta R_{i i}(x, r) \sim \int_{0}^{l} \varepsilon^{2 / 3} \hat{r}^{-4 / 3} \hat{r} d \hat{r} \sim \varepsilon^{2 / 3} l^{2 / 3} \ldots
$$

where $\hat{r}=\left|\left(\boldsymbol{r}^{\prime}-\boldsymbol{r}^{\prime \prime}\right)\right|,|\boldsymbol{r}|=l,\left|\boldsymbol{r}^{\prime}-\boldsymbol{r}^{\prime \prime}\right|<L_{x}, \overline{\omega^{\prime}{ }_{k}\left(\boldsymbol{r}^{\prime}\right) \omega^{\prime}{ }_{l}\left(\boldsymbol{r}^{\prime \prime}\right)} \sim \varepsilon^{2 / 3} \hat{r}^{-4 / 3}$

It can be seen from eq. (5) that although the co-relation of vorticities is significant at small separations of $\hat{r}$, the contribution from smaller-scale vorticity is comparable to that from lengthscales of order $l$. Thus, the contribution to the eddies could come from either vortex sheets separated by $L_{x}$ or from smooth distributions of vorticities on a scale $l$ or from both.

The effect of non-linear terms (4) are to be estimated over the appropriate length scale $(l)$ of the velocity field and the time period $(\mathrm{T})$ over which the distortion is applied. The second term of eq. (4) related to the stretching of the fluctuating vorticity by the fluctuating velocity is of the order $\varepsilon^{1 / 3} l^{-2 / 3}$. Over the time period $\mathrm{T}$, the relative change in the linear and non-linear terms of the vorticity w.r.t. the initial vorticity $\omega_{0}$, is given by $\Delta \omega_{\mathrm{Lin}} / \omega_{0} \sim(\Delta \mathrm{U} / \mathrm{L} \vartheta) \mathrm{T} \vartheta$, where $\Delta \mathrm{U}$ is the change in mean velocity and $\Delta \omega_{\mathrm{NL}} / \omega_{0} \sim\left(\mathrm{u}^{\prime}(l) / l\right) \mathrm{T}$, where $\mathrm{L} g$ is the length-scale of domain $\mathscr{O}$. The criterion for ignoring the nonlinear vortex stretching term is therefore given by the following equation. 
$\frac{u^{\prime(l)}}{l} \sim \varepsilon^{\frac{1}{3}} l^{-\frac{2}{3}} \ll \max \left(\frac{\Delta U}{L_{0}}, \frac{1}{T_{2}}\right) \ldots$

In terms of the characteristic velocity of the energy containing eddies $u^{\prime}$, the inertial range scaling $\mathrm{u}(l) \sim \mathrm{u}^{\prime}{ }_{0}\left(l / \mathrm{L}_{\mathrm{x}}\right)^{1 / 3}$, eq. (6) can be reframed as

$\frac{u_{0}^{\prime}}{L_{x}}\left(\frac{l}{L_{x}}\right)^{-2 / 3}<<\max \left(\frac{\Delta U}{L_{y}}, \frac{1}{T_{2}}\right)$

The total strain defined by $\beta=T, \Delta U / L$ and the relative rate of strain defined by

$$
\mathscr{S}^{*}=(\Delta \mathrm{U} / L) T_{L}, \text { where } T_{L}=\frac{L_{x}}{u_{0}} \ldots
$$

are the two dimensionless quantities characterizing the energy containing eddies. It can be concluded that for weaker strain rates $\mathscr{S}^{*} \leq 1$,

$\left(\beta / \mathscr{S}^{*}\right) \ll 1$, or $T_{\mathscr{O}} \ll T_{L}$

In case the strain rate is strong, $\mathscr{S}^{*} \geq 1$, then

$$
\left(\beta / \mathscr{S}^{*}\right) \gg 1 \ldots
$$

implying $T_{J} / T_{L}$ is arbitrary. Satisfaction of eq. (7) implies that the effects of random straining with large time scales tend to be negligible. Thus, this condition is the essential criterion for the RDT to be valid in case of rapidly changing turbulent flows. Thus, the linearization can be justified only if the strain-rate is significantly large or the period of distortion is significantly short.

The anisotropy caused by the mean strain can be under-estimated or over-estimated by linearization. Therefore, the criterion of eq. (7) which enables us to neglect the non-linear terms is applicable to velocity and vorticity only if modified to take into account the reduce straining in some direction and the nonlinear rotation. This modifies the equation to

$$
\frac{u_{0}}{L_{x}}\left(\frac{l}{L_{x}}\right)^{-2 / 3}<<\max \left(\frac{\Delta U}{L_{0}} \theta\left(T_{\ominus}\right), \frac{1}{T_{0}}\right) \ldots
$$

where, $\theta\left(T_{\partial}\right)=\exp \left(\left(\lambda_{\max }-\lambda_{\min }\right) T_{\partial}\right)$ where $\lambda_{\max } \& \lambda_{\min }$ are the moduli of the maximum and minimum values of principal strain of the mean flow field $\partial U_{i} / \partial x_{j}$. Thus, eq. (10) condition for changes to $\mathscr{S} * \theta \geq 1$. Batchelor [2] proved that for strong enough compressive strains, $\partial U_{i} / \partial x_{j} \alpha \delta_{i j}$ and $\theta=1$, and if the criteria is satisfied, the non-linear terms can be neglected for all time. However, if for any non-isotropic strain the value of $\theta$ increases with time, the non-linear term can no more be neglected.

\section{Summary}

In the present project, we are focusing on the application of the RDT to model the turbulence for the wind farms. The linearization of Navier-Stokes equation using RDT and the errors associated are to be analysed in greater details in this context. Simply ignoring the non-linear terms is of no good if it leads to a great level of approximation errors. Choosing the relevant length scale and time scales of the vortices will play an important role in the approximation using RDT. 


\section{References}

[1] G.K. Batchelor, I. Proudman, The effects of Rapid Distortion of a fluid in turbulent motion, Q. J.Mech. Appl. Maths, 7 (1954), 83-103. https://doi.org/10.1093/qjmam/7.1.83

[2] G.K. Batchelor, The effective pressure exerted by a gas in turbulent motion, In Vistas in Astronomy (ed. A.Beer), 1(1955), 290-295. https://doi.org/10.1016/0083-6656(55)90038-6

[3] G.K. Batchelor, I. Proudman, The large-scale structure of homogeneous turbulence, Phil. Trans. R. Soc. Lond.,A 248(1956), 369-405. https://doi.org/10.1098/rsta.1956.0002

[4] J.C.R. Hunt, D.J. Carruthers, Rapid Distortion Theory and the 'problems' of turbulence, J. Fluid Mech. 212 (1990) 497-532. https://doi.org/10.1017/S0022112090002075

[5] G.K. Batchelor, An Introduction to Fluid Dynamics, Cambridge University Press, 1967

[6] A.S.Monin, A.M.Yaglom, Statistical Theory of Turbulence, Vol. I, MIT Press, 1971.

[7] A.S. Monin, A.M. Yaglom, Statistical Theory of Turbulence, Vol. II, MIT Press, 1975. 